\title{
Stability and succession of the rhizosphere microbiota depends upon plant type and soil composition
}

\author{
Andrzej Tkacz ${ }^{1,2}$, Jitender Cheema ${ }^{1,3}$, Govind Chandra ${ }^{1}$, Alastair Grant ${ }^{4}$ and Philip S Poole ${ }^{1,2}$ \\ ${ }^{1}$ Department of Molecular Microbiology, John Innes Centre, Norwich Research Park, Norwich, UK; \\ ${ }^{2}$ Department of Plant Sciences, Oxford University, Oxford, UK; ${ }^{3}$ Department of Computational and Systems \\ Biology, John Innes Centre, Norwich Research Park, Norwich, UK and ${ }^{4}$ Earth and Life Systems Alliance, The \\ School of Environmental Sciences, University of East Anglia, Norwich, UK
}

\begin{abstract}
We examined succession of the rhizosphere microbiota of three model plants (Arabidopsis, Medicago and Brachypodium) in compost and sand and three crops (Brassica, Pisum and Triticum) in compost alone. We used serial inoculation of 24 independent replicate microcosms over three plant generations for each plant/soil combination. Stochastic variation between replicates was surprisingly weak and by the third generation, replicate microcosms for each plant had communities that were very similar to each other but different to those of other plants or unplanted soil. Microbiota diversity remained high in compost, but declined drastically in sand, with bacterial opportunists and putative autotrophs becoming dominant. These dramatic differences indicate that many microbes cannot thrive on plant exudates alone and presumably also require carbon sources and/or nutrients from soil. Arabidopsis had the weakest influence on its microbiota and in compost replicate microcosms converged on three alternative community compositions rather than a single distinctive community. Organisms selected in rhizospheres can have positive or negative effects. Two abundant bacteria are shown to promote plant growth, but in Brassica the pathogen Olpidium brassicae came to dominate the fungal community. So plants exert strong selection on the rhizosphere microbiota but soil composition is critical to its stability. microbial succession/ plant-microbe interactions/rhizosphere microbiota/selection.
\end{abstract}

The ISME Journal (2015) 9, 2349-2359; doi:10.1038/ismej.2015.41; published online 24 April 2015

\section{Introduction}

The rhizosphere is a critical zone of soil surrounding roots that is directly influenced by the plant and into which plants secrete as much as $11 \%$ of net fixed carbon (Jones et al., 2009; Dennis et al., 2010). It is a 'hot spot' of microbial activity, with increased microbial numbers, microbial interactions and genetic exchange (Bulgarelli et al., 2013; Turner et al., 2013a). There is a close two-way interaction between the microbial community (microbiota) and plant host that is an essential determinant of plant health and productivity. This is very apparent in symbiotic associations between rhizobia and legumes, resulting in fixation of $40-50 \%$ of the biosphere's nitrogen (Terpolilli et al., 2012; Udvardi and Poole, 2013) as well as widespread acquisition of nutrients such as phosphate by mycorrhizae (Lanfranco and Young, 2012; Nuccio et al., 2013).

Correspondence: PS Poole, Department of Plant Sciences, University of Oxford, South Parks Road, Oxford, OX1 3RB, UK. E-mail: Philip.poole@plants.ox.ac.uk

Received 6 June 2014; revised 5 February 2015; accepted 16 February 2015; published online 24 April 2015
However, interaction between plants and microbes is more general with plants shown to shape the rhizosphere microbiota in legumes, potatoes and suppressive soils (Sharma et al., 2005; Lebreton et al., 2007; Manter et al., 2010; Kinkel et al., 2011; Mendes et al., 2011; Turner et al., 2013b). Some microbial root colonists are saprophytes, which also colonise splinters of wood inserted into soil (Bulgarelli et al., 2012), but others are selected by exudation of nutrient sources and phytoalexins, such as glucosinolates and avenacins (Sonderby et al., 2007; Bressan et al., 2009; Turner et al., 2013b). Furthermore, by altering the rhizosphere microbiota, plants induce formation of suppressive soil where growth of plant pathogens is inhibited (Lebreton et al., 2007; Kinkel et al., 2011; Mendes et al., 2011). Microbes can increase plant growth by releasing phytohormones, biofertilization (Lugtenberg and Kamilova, 2009) and stimulation of both the induced systemic resistance and systemic-acquired resistance components of the plant immune system (Van der Ent et al., 2009). It has been difficult to study these complex interactions at the community level but recently high-throughput sequencing methods have been used to show that Arabidopsis preferentially 
selects specific microbial endophytes, which colonise plant tissues, with some, albeit weaker, selection in the rhizosphere (Bulgarelli et al., 2012; Lundberg et al., 2012).

Previous studies on the soil microbiota of plants grown in natural soil have shown that plant and soil are both important factors in community structure (Berg and Smalla, 2009). However, as shown by Lauber et al., 2009, soil pH also has a dramatic influence on the soil microbiota. Studying field grown plants for their influence on the microbiota is hindered by high soil complexity and local variation in $\mathrm{pH}$. Moreover, most previous studies used DNA fingerprinting methods with limited replication $(<4$ samples). As we show here it is vital to sample at least 10-15 rhizosphere samples (biological replicates) in order to establish statistical significance.

To study microbial succession we used a nutritionally defined medium that in the first generation consisted of a $10 \%$ soil inoculum mixed with either autoclaved sand or compost. In two succeeding generations, $25 \%$ of the growth medium was transferred from the preceding generation and mixed with $75 \%$ sand or compost. This allowed control of soil nutrients, as they were refreshed in each generation and separation of the effects of soil characteristics from those of the initial microbial community composition. This approach was used to examine rhizosphere succession over three generations in three model and three crop plants, belonging to distinct families. Sand and compost were used to allow assessment of the relative importance of root exudation and soil organic matter in supporting rhizosphere communities.

\section{Materials and methods}

Soil collection and plant growth

Soil used as a microbial inoculum was collected from a naturally grassed and unfertilised part of John Innes Centre Church Farm, Bawburgh, Norfolk, UK $\left(52^{\circ} 37^{\prime} 39.35^{\prime \prime} \mathrm{N}, 1^{\circ} 10^{\prime} 43^{\prime \prime} \mathrm{E}\right)$. Covering vegetation was stripped off and soil collected from a depth of 10-30 cm. Chemical analyses (by MacaulaySoils, James Hutton Institute, Aberdeen, UK) showed that the soil is poor in nutrients $\left(\mathrm{NO}_{3}^{-} 3.49 \mathrm{mg} \mathrm{kg}^{-1}, \mathrm{P}^{-3}\right.$ $120.5 \mathrm{mg} \mathrm{kg}^{-1}, \mathrm{~K}^{+} 168.2 \mathrm{mg} \mathrm{kg}^{-1}, \mathrm{Mg}^{2+} 33.55 \mathrm{mg} \mathrm{kg}^{-1}$ ), is $\mathrm{pH} 7.5$ and contains $2.92 \%$ organic matter. According to the UK Soil Observatory, the soil can be classified as Luvisol (WRB classification) and the soil texture was loamy with bits of chalk in the layer below the top layer. This soil was chosen as the history of its plant coverage is well documented and the microbial communities have been characterised in a previous study (Turner et al., 2013b).

Seeds were surface sterilised by using ethanol $(70 \%$ or $95 \%)$ and bleach $(2-10 \%)$ and germinated on MS medium (Murashige and Skoog, 1962) containing essential macro- and microelements for plant growth and sucrose $(3 \%)$. For further details of the seed sterilisation method see Supplementary Table S1. Only seedlings coming from non-contaminated MS plates were planted. We assumed that the seed endophytic community is negligible compared to the soil community as already shown (Lundberg et al., 2012). Plants were grown in a growth room in closed containers in $12 \mathrm{~h} / 12 \mathrm{~h}$ light/dark conditions at $23^{\circ} \mathrm{C}$. Model plants (Arabidopsis thaliana Col-0, Medicago truncatula Jemalong A17 and Brachypodium distachyon Bd21) were grown in 50-ml sealed pots and crop plants (Brassica rapa R-O-18, Pisum sativum cv. Avola and Triticum aestivum cv. Paragon) in $100 \mathrm{ml}$ sealed pots. Two artificial soils were used; poor soil consisting of silver sand (washed, lime free, horticultural grade, manufactured by J Arthur Bower's, Sinclair, Lincoln, UK) and referred to as 'sand', and a nutrient rich, commercially available compost (Everris Levington F2 Seed \& Modular, Scotts, Ipswich, UK) referred to as 'compost' and containing $144 \mathrm{mg} \mathrm{l}^{-1}$ total nitrogen, $73 \mathrm{mg} \mathrm{l}^{-1}$ total phosphorous and $239 \mathrm{mgl}^{-1}$ total potassium (manufacturer's analysis). Example photographs of the plant growth are presented in the Supplementary Word file 1. Sand and compost were autoclaved and tested for any residual microorganisms using TY medium. Bacteria were not recovered although compost still possessed viable fungal spores. To each artificial soil a well-mixed Bawburgh soil inoculum was added and mixed well (10\% vol/vol) (Figure 1). Plants in sand were watered once a week with plant mineral supplement (Poole et al., 1994b). $\mathrm{KNO}_{3}$ (10 mg per pot) was added to each pot of sand at the start of week one of each generation. No nutrients were added to compost, as it is already a rich medium.

Plants were grown for 4 weeks over three generations from a $10 \%$ inoculum of Bawburgh soil. For generations two and three, $25 \%$ (vol/vol) of the growth medium from the preceding generation was mixed with either $75 \%$ sand or compost (Figure 1). The starting soil inoculum was diluted over successive generations so the community would increasingly depend on root exudates as a carbon source, especially in sand. With the alternative experimental strategy of replanting in the initial soil, changes in microbiota could be caused by gradual reductions in the nutrient status of the growth medium. In total, 24 independent replicates for each plant species and unplanted control were maintained. At harvest, the shoot was removed and root shaken to remove loosely adhering soil. Soil left in the pot (and fine fragments of roots, which could not be removed) was used as a microbial inoculum for further generations. Because of the small pot size all the soil in the pot was influenced by root exudates (Supplementary Word file 1). Roots were placed in a Falcon tube $(50 \mathrm{ml})$ with phosphate buffered saline $(30 \mathrm{ml})$ and vortexed (30 s) until the root was visibly clear from adhering soil, before roots were discarded and the soil suspension centrifuged at an RCF of 2880 for 5 min (Centrifuge 5810, Eppendorf, Stevenage, UK). 


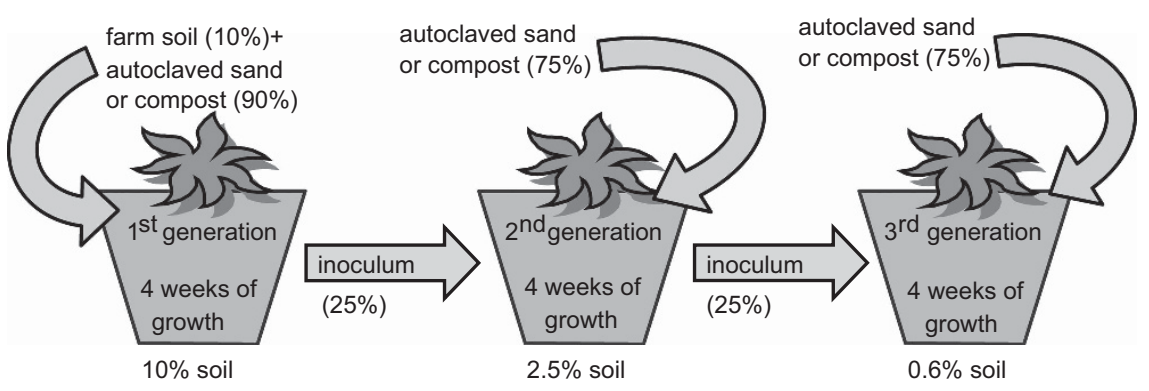

Figure 1 Experimental design of multi-generation plant growth. Autoclaved sand or compost (90\% by volume) was inoculated with Bawburgh grass soil ( $10 \%$ by volume) for the first generation of plant growth. In generations two and three, soil ( $25 \%$ by volume) from the previous generation was used as an inoculum. Bawburgh grass soil constituted 10, 2.5 and 0.6\% (volume) in successive generations. Identical treatment was given to unplanted controls for three generations. After 4 weeks growth plants were harvested and DNA isolated from the rhizosphere for ARISA and 454Flx analysis of the microbiota.

The supernatant was decanted and DNA was isolated from $1 \mathrm{~g}$ (wet weight) of rhizosphere soil (Griffiths et al., 2000) with humic acids removed by using the ZYMO research One-step PCR inhibitor removal kit (Zymo Research, Irvine, CA, USA).

\section{Isolation of soil bacteria}

To isolate soil microorganisms three different growth media were used: LB (Bertani, 1951), TY (O'Reilly and Niven, 1986) and acid minimum salts agar (Poole et al., 1994a) supplemented with glucose (10 mM) and ammonia (10 mM). Rhizosphere soil (1 g) was mixed with acid minimum salts agar (no carbon or nitrogen source added) and shaken for $15 \mathrm{~min}$, centrifuged at low speed to pellet the soil particles, the supernatant was transferred to a fresh tube and centrifuged at an RCF of 2880 for $5 \mathrm{~min}$. The supernatant was discarded and the microbial pellet diluted in acid minimum salts agar $(5 \mathrm{ml})$ and serially diluted on Petri plates containing LB, TY or acid minimum salts agar (Supplementary Word file 2).

\section{Plant growth promotion assay}

Seeds of Arabidopsis, Medicago and Brachypodium (24 replicates) were surface sterilised as above. Plants were grown in sterilised silver sand and rooting solution and nitrogen added to the same levels given above. Seedlings were inoculated after the first week of growth with either Achromobacter xylosoxidans or Arthrobacter sp. or left uninoculated. Bacteria were grown overnight in the liquid culture (TY) and the plants inoculated with $10^{8}$ of viable cells (calculated by optical density measurement and confirmed by cell counting). Bacteria were spun down, washed free of media, re-suspended in water and applied to plant roots. Plants were grown for 4 weeks before the shoot was cut-off, dried for $48 \mathrm{~h}$ at $70^{\circ} \mathrm{C}$ and weighed.

\section{Data analysis}

Community structure was analysed by Automated Ribosomal Intergenic Spacer Analysis (ARISA) (Fisher and Triplett, 1999) by using fluorescently labelled ITSF (5'-GTCGTAACAAGGTAGCCGTA-3') and ITSReub (5'-GCCAAGGCATCCACC-3') primers and PCR conditions described already (Cardinale et al., 2004). 454Flx titanium pyrosequencing was performed at The Genome Analysis Centre, Norwich, UK with DNA from 24 individual plants binned into three pools of 8 . For 454Flx sequencing the bacterial 16S rDNA (V1-V2 fragment) was amplified by using 27F and 338R primers and PCR conditions described already (Hamady and Knight, 2009). The fungal ITS region was amplified using ITS1 and ITS2 primers and PCR conditions described already (Buee et al., 2009). Bacterial and fungal reads were separated by the primer sequence. Additional information about sequencing is provided in Supplementary Table S2.

Reads were de-noised, quality filtered and the 454 adapters removed using a Roche (Branford, CT, USA) data pipeline at The Genome Analysis Centre. The reads were split based on the PCR primers used (bacteria or fungi) and the barcode from the Roche MID adapters library. The total pool of reads was searched by custom-made script looking for a $100 \%$ match of the PCR primer and the barcode. All the reads with any mismatch or a gap in either PCR primer sequence or the barcode were removed. This approach selected for reads with both PCR primer sequences intact, so all the reads had the right length (there can be some small difference in the V1-V2 region of 16S rRNA (in case of bacteria) or ITS (in case of fungi) fragment itself, which either arose as a sequencing errors or are truly biological differences in this region length between different bacterial/ fungal species). Our approach in combination with the initial analysis at the genomic centre provided us with high-quality reads ready for the downstream analyses. Screening for chimeras was initially carried out by using DECIPHER (Wright et al., 2012). However, this gave a number of apparent false positives. These were relatively common (up to $0.5 \%$ of reads), occurred in several replicate samples, and matched a number of $16 \mathrm{~S}$ sequences in the NR Genbank database at $>95 \%$ similarity, including matches to close to full length $16 \mathrm{~S}$ sequences, which in some cases, were from cultured strains of Rhizobium and Bradyrhizobium. So we adopted an alternative approach of removing from each data set 
Operational Taxonomic Units (OTUs) that represented $<0.1 \%$ of reads. This removed all sequences identified as chimeric by DECIPHER (as well as other rare sequences) but retained the 'false positives' referred to above.

Sequences were binned into OTUs by using USearch 6.0 (drive5) (Edgar 2010), set at 95\% sequence identity between reads (Supplementary Table 2) (Wooley et al., 2010). Bacterial 16S rRNA gene reads were aligned against SILVA (v.115; Max Planck Institute for Marine Microbiology, Bremen, Germany) and fungal ITS reads were aligned against the full Genbank database. OTUs were annotated with MEGAN software (University of Tübingen, Tübingen, Germany), using the default settings, apart from the settings in the Last Common Ancestor (min support = 1 and top percent=1) (Huson et al., 2009).

The fluorescence intensity of ARISA peaks and abundance of OTUs were exported to Microsoft Excel, aligned into size bins of $0.8 \mathrm{bp}$. using T-Align (Smith et al., 2005) and exported into Primer 6.0 (PRIMER-E, Ivybridge, UK) and R (R Development Core Team, 2008) for analysis. Each value was standardized (as a \% of the total), square root transformed and a similarity matrix constructed using the Bray-Curtis coefficient. Results were visualised by using non-metric multi-dimensional scaling. Differences between groups were tested for significance using a permutation based analysis of variance (multivariate analysis of variance) by using the adonis function of the vegan package in $\mathrm{R}$ (Osborne et al., 2011), including plant species and generation as factors plus the interaction between them.

3D ternary plots were generated by using a bespoke Python (2.6; Python Software Foundation, Beaverton, OR, USA) script and the diagrams drawn by using Jmol (13.0.15, an open-sourve Java viewer for chemical structures in 3D; http://www.jmol.org/) under Mac OS X (10.6.4, Apple, Cupertino, CA, USA) and was further annotated using Adobe Illustrator (CS5, Adobe Systems, San Jose, CA, USA). The script used is freely available from the authors on request.

Representative reads from all individual OTUs were aligned by using ClustalW (EMBL-EBI, Hinxton, UK) and imported into MEGA5 (Centre for Evolutionary Medicine and Informatics, Tempe, AZ, USA) to produce a dendrogram. Based on the dendgrogram, the OTUs were placed according to their phylogenetic relation and their ratio of abundance in the rhizosphere against unplanted control (calculated for each generation) visualised using heat maps. These maps were constructed using the online tool matrix2png (http:// www.chibi.ubc.ca/matrix2png/). The Shannon index of diversity and community richness were calculated in Microsoft Excel for 454Flx data.

\section{Results}

Experimental selection system

Our experimental selection system allows us to examine the influence of plants on the rhizosphere microbiota over several generations. Two sterile artificial growth media were used (see Materials and methods); silver sand with complete mineral solution added (sand) and a commercial compost rich in nutrients and organic material (compost). The starting microbiota (inoculum) was introduced by adding 10\% Bawburgh soil to both artificial, sterilised growth media (sand or compost). An inoculum of $10 \%$ was chosen to minimise the influence of the soil inoculum on the nutrient status of the growth medium. These soils were low in infective rhizobia and neither Medicago nor Pisum were nodulated in any of the experiments reported below. However, in a separate test, nodules formed when appropriate rhizobia were added (data not shown). No mycorrhization was observed (data not shown). Bawburgh soil inoculum was only used to prepare the initial plant growth medium (generation 1) and successive generations were started using the same growth medium (either sand or compost) and microbial inoculum from the preceding generation $(25 \%$ of the volume). A volume of $25 \%$ was used to ensure that the microbial community was transferred efficiently, while minimising the stochastic loss of rarer species (Bent and Forney, 2008). The alternative approach of growing plants in the same soil could lead to depletion of nutrients.

\section{Microbiota succession in sand}

For clarity we first consider the microbiota of plants grown in sand. To check the stability of the starting inoculum over time, Arabidopsis plants were grown by using Bawburgh soil inocula (10\% in sand) freshly sampled more than 1 year apart (November 2009 and July 2011). There were no major differences, apart from the absence of Achromobacter in the second year, in the abundance of the 10 most common bacterial OTUs or the Shannon diversity index (Supplementary Figure S1), showing that the starting inocula were very stable.

To begin the succession experiment, Arabidopsis, Medicago and Brachypodium (a model non-legume dicot, legume and monocot cereal, respectively) were grown in sand with a common Bawburgh soil inoculum. After only one generation of growth, there was a strong effect, with each plant's microbiota differing from all others and from the unplanted soil (most readily visible in the bacterial ARISA data where replication is greatest Figure 2a). The microbiota of all plants and unplanted soil samples show a similar shift in composition over generations, with points representing the first generation lying towards the left of Supplementary Figures S2a and d, and those representing the third generation towards the right. The microbiota of each plant species in each generation are clustered together, as are the microbiota of unplanted soil samples, with the unplanted microbiota usually at the periphery of the distribution of points in each generation, showing the effect of plant selection. The differences between times were 
greater than those between plants (Table 1), although the effects of both, and the interaction between them were all significant. We hypothesise that a considerable component of this change over time is caused by a natural Bawburgh soil community adapting to soil dilution over three generations. Moreover the environmental conditions (for example, temperature, watering) differ greatly between the original soil (to which the community was adjusted) and the controlled laboratory conditions. Multivariate analysis of

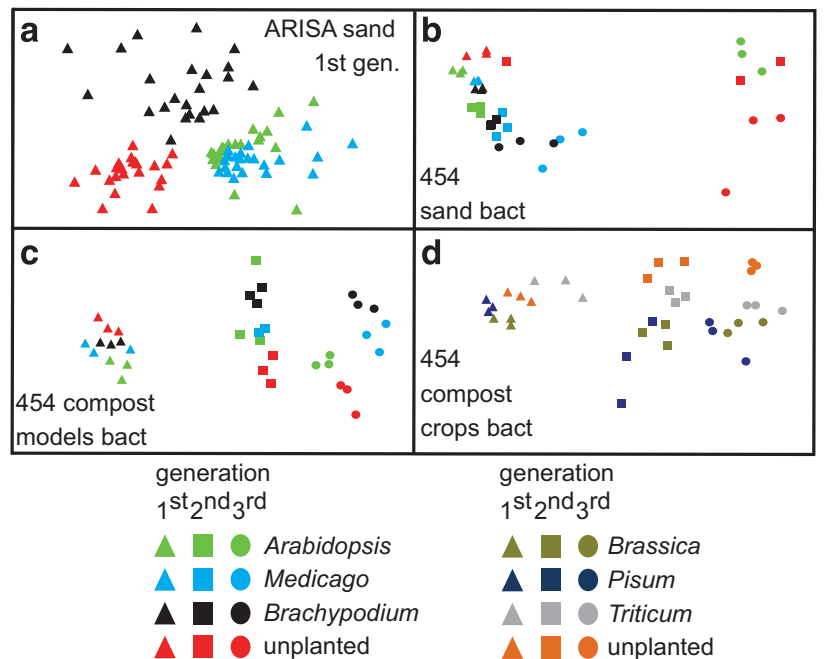

Figure 2 Different model and crop plants grown in sand and compost establish distinct rhizosphere microbiota. (a) Sand: ARISA analysis of first generation of plants. (b) Sand: 454Flx pyrosequencing analysis of bacteria from all three generations. (c) Compost: 454Flx pyrosequencing analysis of bacteria from three generations of model plants. (d) Compost: 454Flx pyrosequencing analysis of bacteria from three generations of crop plants. Data shown as $n$ multi-dimensional scaling plots (based on Bray-Curtis dissimilarity), which lack axes and where each data point represents one plant microbiota.

Table 1 Percentage of variance in microbiota community composition explained by plant species, generation and the interaction between them for ARISA and 454 data

Species Generation Interaction Residual

\begin{tabular}{lcccc}
\hline $\begin{array}{l}\text { ARISA } \\
\text { bacteria-compost }\end{array}$ & 12 & 20 & 14 & 54 \\
$\begin{array}{l}\text { ARISA } \\
\text { bacteria-sand }\end{array}$ & 11 & 28 & 10 & 52 \\
$\begin{array}{l}454 \\
\text { bacteria-compost }\end{array}$ & 18 & 39 & 16 & 26 \\
$\begin{array}{l}454 \\
\text { Bacteria-sand }\end{array}$ & 27 & 34 & 20 & 18 \\
$\begin{array}{l}454 \\
\text { Fungi-compost }\end{array}$ & 29 & 23 & 21 & 27 \\
$\begin{array}{l}45 \\
\text { Fungi-sand }\end{array}$ & 13 & 26 & 17 & 44 \\
\hline
\end{tabular}

Abbreviation: ARISA, Automated Ribosomal Intergenic Spacer Analysis.

All effects are significant at $P<0.001$, except for the interaction term for fungi in sand, where $P=0.003$. For compost experiments, all six plant species and both unplanted controls are analysed simultaneously. Sand experiments used only model plants. variance performed on the sand experiment confirms the multi-dimensional scaling observations, that the differences between the rhizospheres and unplanted soil are real (Supplementary Figure S3).

ARISA enabled large numbers of replicate plants to be tested ( $\sim 24$ for each generation), and provides high taxonomic resolution due to variability of the bacterial 16S-23S rDNA intergenic spacer region (Ngugi and Stingl, 2012), but only a fingerprint of community structure. Therefore high-throughput 454Flx pyrosequencing of the bacterial V1-V2 $16 \mathrm{~S}$ rDNA or fungal intergenic spacer region was used to group the rhizosphere microbiota into OTUs. OTU data from $454 \mathrm{Flx}$ sequencing confirmed that plant rhizosphere microbiota diverged strongly from each other and over generations. They also confirmed that Arabidospsis in the third generation was closer to unplanted soil than either Medicago or Brachypodium (Figure 2b), suggesting Arabidopsis biased the rhizosphere microbiota less than the other plants. The fungal microbiota followed a similar pattern with rhizosphere communities close together in the first generation but more divergent in the second and third generations (Supplementary Figure 2g). The 454 data showed less variation between replicates than did ARISA, with the two factors and their interaction explaining between $56 \%$ and $82 \%$ of the total variance (Table 1), probably because DNA from eight individual plants was pooled into each replicate before pyrosequencing.

Poor soil conditions over successive generations favour fast growing Proteobacteria (Lambert et al., 1990; Demoling et al., 2007), consistent with some groups becoming increasingly dominant in generations two and three (Supplementary Figure S4a). The most abundant bacterial OTUs selected were $\alpha$-proteobacteria (Rhizobiaceae family), $\beta$-proteobacteria (especially the genus Massilia), and Bacteroidetes, whereas the second most abundant group, the actinobacteria, decreased in the rhizosphere over generations (Supplementary Figures S5a and b). Selection is displayed most dramatically in ternary plots (see Supplementary Figure S6 for an explanation), where each sphere represents a single OTU, size represents abundance, position corresponds to the relative abundance in the three rhizospheres and colour reflects the rhizosphere abundance relative to unplanted soil (Figure 3 and Supplementary Figure S7). Sphere colour represents is based on the relative abundance (rhizosphere/unplanted control) of a particular OTU for each generation. Thus, bluegreen OTUs are primarily soil organisms, whereas orange-red OTUs are rhizosphere specific. A few bacterial and fungal OTUs, that are abundant and/or of special interest are annotated (Figure 3 and Supplementary Figure S7). In the first generation, there were some generalist- (red spheres in the middle) and plant-specific rhizosphere OTUs (red spheres in the corners), but the majority were found in both rhizosphere and soil (green and yellow spheres). However, in the second generation, most OTUs are 

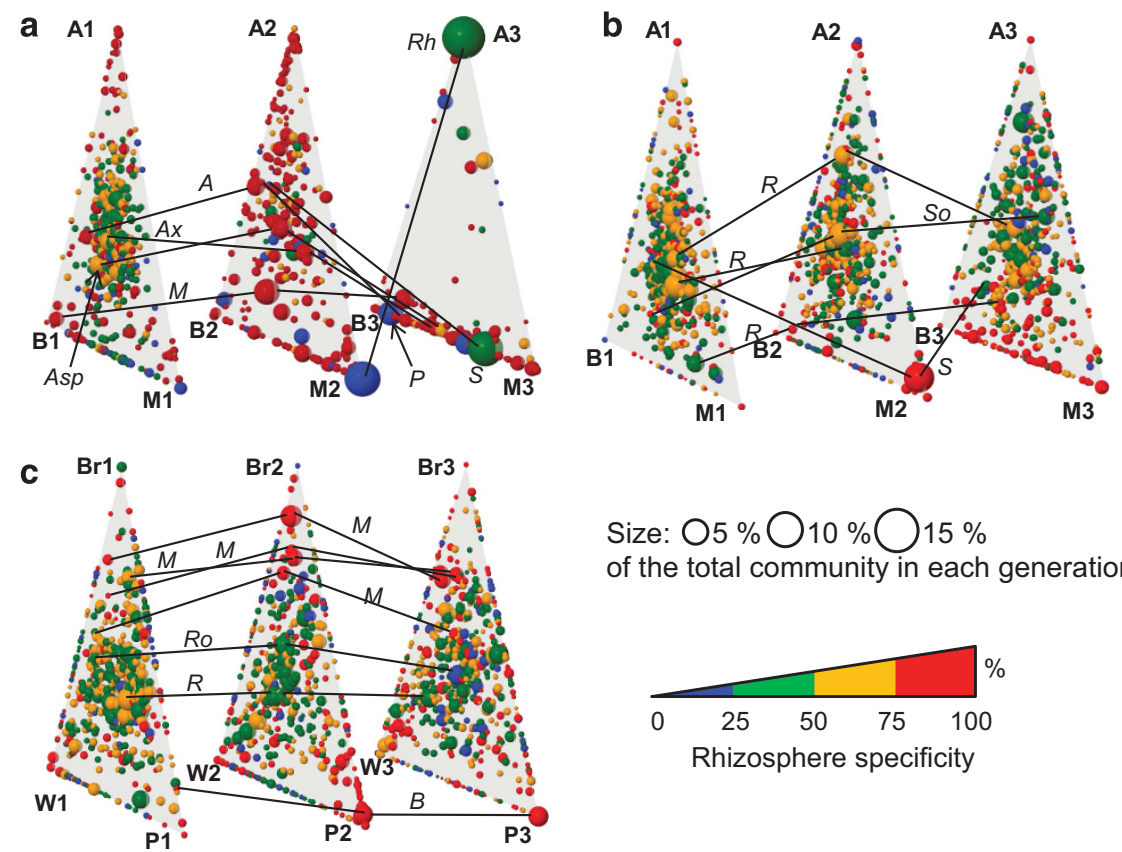

Size: $05 \% \bigcirc 10 \% \bigcirc 15 \%$

of the total community in each generation

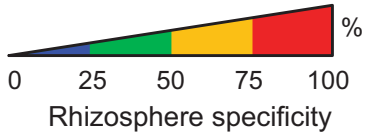

Figure 3 The effect on the bacterial rhizosphere microbiota of plants grown in sand compared with compost. Each sphere represents a single OTU, size represents abundance, position corresponds to the relative abundance in the three rhizospheres and colour reflects the rhizosphere abundance relative to unplanted soil calculated for each generation separately. Please refer to Supplementary Figure S6 for an example explanation. (a) Bacterial microbiota of model plants grown in sand. (b) Bacterial microbiota of model plants grown in compost. (c) Bacterial microbiota of crop plants grown in compost. Only OTUs with a relative abundance of greater than $0.1 \%$ were used. Achromobacter xylosoxidans (Ax), Acidovorax (A), Arthrobacter sp. (Asp), Massilia (M), Pseudomonas (P), Rhizobium (three separate OTUs are annotated) (R), Rhodopseudomonas (Rh), Rodanobacter (Ro), Solirubrobacter (So) Stenotrophomonas (S) and Arabidopsis (A), Medicago (M), Brachypodium (B), Brassica (Br), Pisum (P), Triticum (W) and generation (1, 2, 3).

red, indicating a greater abundance in the rhizosphere. In the third generation, the diversity of the Arabidopsis microbiota collapsed, with invasion of apparent opportunists that were also common in unplanted soil (green and blue spheres) (Figure 3a). The dominant bacterial species in the third generation were predominantly putative autotrophs such as Rhodopseudomonas in Arabidopsis and Stenotrophomonas in Medicago and Brachypodium (Figure 3a). Isolates of these species are either able to fix $\mathrm{CO}_{2}$ and gain energy from photosynthesis and use nitrate as an electron acceptor (present in plant mineral supplement) or fix $\mathrm{N}_{2}$ from the atmosphere (Taghavi et al., 2009; Yuan et al., 2012). However, the fact that the dominant opportunists present in the third generation of Arabidopsis were different from Medicago and Brachypodium suggests that plant selection still operated. The putative autotrophic lifestyles of these opportunists may give an advantage over species utilising plant-derived compounds and/or soil residues. This is particularly the case when the percentage of Bawburgh soil in sand decreased from $10 \%$ to $2.5 \%$ to $0.6 \%$ over the three generations, with a commensurate reduction in organic material (Figure 1). Brachypodium and Medicago grown in sand also showed a reduced diversity, although to a lesser extent than for Arabidopsis (Supplementary Figure S4b). A similar reduction in diversity in the third generation also occurred for fungi (Supplementary Figures S4b and S8a).
Plants select for plant growth promoting bacteria At the end of the third generation we isolated soil bacteria from rhizosphere samples. One advantage of this experimental selection experiment is that 454Flx analyses revealed the abundance of different OTUs for each plant in each generation. Thus it was known that the genera Achromobacter, Arthrobacter and Massilia were among the most abundant and strongly selected bacteria. To see if these could be isolated, 150 bacterial strains from the third generation of Arabidopsis, Medicago and Brachypodium were cultured (50 isolates from each plant species) using different growth media and individual colonies were screened by using 16S rRNA sequencing (Supplementary Word file 2). Achromobacter xylosoxidans and Arthrobacter sp. but not Massilia were successfully isolated (annotated as Ax and Asp on Figure 3a, respectively). A. xylosoxidans and $A$. sp were isolated from Medicago and Brachypodium rhizospheres, respectively. The corresponding OTUs appear to be soil borne bacteria as they were also found at low abundance in the unplanted control samples (Supplementary Figure S9). While both organisms were present in soil they were strongly selected in the rhizosphere apart from the third generation of Arabidopsis. When re-inoculated as pure cultures onto plants grown in sterile sand, Achromobacter increased growth of all plants, while Arthrobacter increased the growth of Brachypodium from which it was initially isolated (Supplementary 
Figure S10). Thus these isolates are plant growth promoting rhizobacteria that form a mutualistic relation with a plant, promoting plant growth and presumably developing a favourable rhizosphere niche for their own growth. Thus while this experimental approach is not intended to replicate natural soil it is a very powerful way to investigate plantmicrobe interactions and select plant-dependent bacteria.

\section{Microbiota selection in compost}

Selection of the rhizosphere microbiota in sand (Figures 2a and b and Supplementary Figures S2a, $\mathrm{d}$ and g) creates conditions in which plant exudate is the dominant organic nutrient source. This makes it particularly suited to identification of the role of plants in selection of microbiota, independent of soil factors. However, it is important to test a more complex soil to compare the effect of both growth media and plant species in selection of microbiota. Therefore the impact of plants on rhizosphere communities over three generations was measured in nutritionally rich compost. Again the microbiota was influenced by plants (Figures 2c, d, 3b and c, Supplementary Figures S2b, c, e, f, h, i, k, S5c, d), with $\alpha$-proteobacteria (particularly the order Rhizobiales) strongly selected. However, actinobacteria increased in abundance in compost. Out of 41 actinobacterial OTUs showing a significant difference between the rhizosphere and unplanted control, 29 were more common in rhizospheres, in contrast to their depletion in sand (out of 38 Actinobacterial OTUs, 24 were depleted in sand; Supplementary Figures S5b and c).

Model plant species are well characterised genetically and have been chosen for their small size and rapid growth. However, these growth habits might be unusual in causing strong effects on their rhizosphere microbiota. We therefore tested larger, widely planted crop plants Brassica, Pisum and Triticum, which belong to the families Brassicaceae, Fabaceae and Poaceae, respectively. Crop plants, grown in compost, had strong shifts in their rhizosphere microbiota over three generations (Figure 2d). Indeed, crop species had a greater influence on the rhizosphere than model plants, selecting more rhizosphere-specific bacterial OTUs (Supplementary Figures S2k, S5c and S11). This is probably explained by the more extensive root systems of crop plants, leading to increased root exudation and root turnover (Hawes et al., 2000).

In addition, for all six species of plants the microbial community was much more stable during growth in compost compared with sand, with no loss of diversity over generations as indicated by the Shannon index of diversity (Supplementary Figures S4c and d). The statistical analysis performed on the richness values (Supplementary Figure S4e) indicates that indeed there is a disturbance in the community of sand and also to some extend in the compost after the initial generation. Moreover, there are clear differences between compost and sand community richness in the following generations. In generation two and three compost maintained a relatively stable microbiota, although the microbiota of Arabidopsis in the third generation split into three very different communities of bacteria, suggesting stochastic variation (Supplementary Figure S2j). Potentially, these sub-communities could diverge further in subsequent generations, leading to formation of microbiota with very different properties. This effect may be the cause of the alterations in growth of Arabidopsis grown over multiple generations observed by Swenson (Swenson et al., 2000).

The fungal microbiota and olpidum brassicae as a hostspecific rhizosphere pathogen

Fungal communities contained many fewer OTUs than the bacterial one (Supplementary Figure S7). In contrast to bacterial $16 \mathrm{~S}$ sequences, many fewer fungal ITS sequences were available to use in taxonomic assignments. As a consequence, most fungal sequences could only be annotated to the division/subdivision level. With the exception of Brassica, all communities were dominated by Ascomycota, particularly Saccharomyceta (Supplementary Figure S7). Each plant species had a distinctive fungal community, with replicates from each generation of each species usually clustering together in the multi-dimensional scaling plots, although the effect of generation was not as strong as in the 454 bacterial data, and there was more variation between replicates in sand (Supplementary Figures S2g and i, and Table 1).

Basidiomycota were more abundant in compost than in sand, but there were few differences in the abundance of divisions and subdivisions between plant species (Supplementary Figure S8). The only large changes were observed for Brassica, where one OTU became steadily more abundant over time, increasing from $6 \%$ to $61 \%$ then to $81 \%$ of the fungal community over generations 1-3, resulting in a dramatic shift of the overall fungal population (Figure 4 and Supplementary Figures S2i and S7C - annotated as ' $\mathrm{O}$ '). The consensus intergenic spacer of this OTU had $100 \%$ identity to Olpidium brassicae (Figure 4 and Supplementary Figures S12 and S7c - annotated as 'O'). O. brassicae is a fungal pathogen abundant in Brassica rhizospheres (Hartwright et al., 2010) that reduces production of pods and seeds (Hilton et al., 2013). The organism in our samples is likely to be $O$. brassicae, or a close relative, although ultimately this would require confirmation by culturing or more extensive sequencing. The taxonomic position of Olpidium is still a matter of debate, but here it was classified into Fungi incertae sedis (other phyla) (Supplementary Figure S8).

This remarkable selection of an OTU with a taxonomic assignment to a pathogen in a susceptible host in the experimental selection system could 
2356

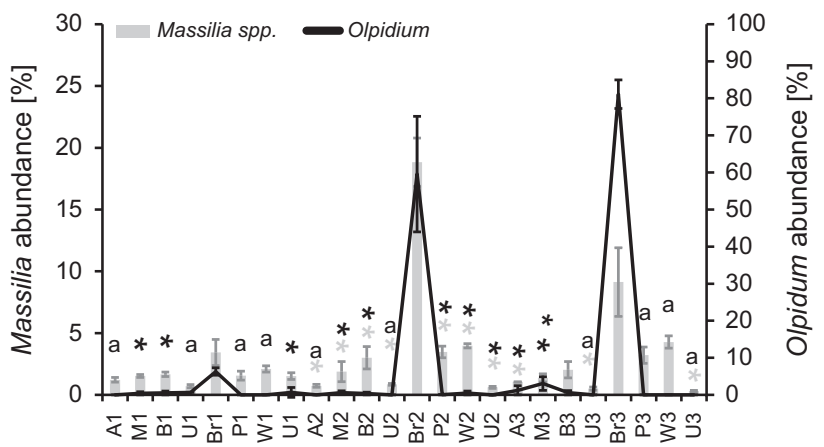

Figure 4 An invasion of Olpidium brassicae on Brassica rapa corresponds with increased abundance of Massilia. The black line represents $O$. brassicae abundance and bars represent abundance of the genus Massilia. B. rapa was grown for three generations on compost, A. thaliana (A), M. truncatula (M), Brassica distachyon (B), B. rapa (Br), Pisum sativum (P), Triticum aestivum (W), unplanted control (U), generation $(1,2,3)$. The abundance of Massilia and Olpidium in Brassica rhizospheres was compared against other rhizospheres using $t$-tests. Grey and black stars represent statistical significance result for Massilia and Olpidium abundance, respectively. ${ }^{*} P<0.05$, a-absent. Error bars represent the standard error calculated from three biological replicates.

mirror the problems encountered with repeated plant monoculture in agriculture. There was also a similar increase in abundance of the bacterial genus Massilia, suggesting it may be related to this invasion. Since this genus grows on chitin as a carbon and nitrogen source (Faramarzi et al., 2009; Adrangi et al., 2010) there may be a causal link, with Massilia growing on material derived from the putative O. brassicae (Figure 4) (James et al., 2006).

\section{Discussion}

We established a system of experimental selection in microcosms by using a defined starting microbial inoculum and controlled soil structure to determine how different plant species and growth media interact to alter succession of the rhizosphere microbiota. Previous studies have compared the influence of plant and soil on the rhizosphere microbiota (Berg and Smalla, 2009), but for the first time we have used high replication to allow statistical analysis to be applied. In addition we compared six different plants and two soil systems to increase the robustness of our observations. Finally, $454 \mathrm{Flx}$ sequencing was used to determine the nature of the organisms selected by different plants. Our two key questions were: how strongly do different plants select the rhizosphere microbiota and how is this influenced by growth media?

With regard to the first question, it is clear that plants select very distinct bacterial and fungal rhizosphere microbiota and this is confirmed by multivariate analysis of variance analysis, the proportion of variance in community composition explained by plant species and its interaction with time (Table 1). While fingerprinting techniques such as ARISA and T-RFLP (terminal restriction fragment length polymorphism) have been used before and suggest plant dependent differences, our data show that to detect differences within a single generation more replicates are required (typically greater than 10) than are usually used (Knight et al., 2012). Crop plants, with more extensive root system, shifted the community more than smaller model species, but all plants tested had very different microbiota from each other and from unplanted soil (Figures 2a and d, and Supplementary Figure S2).

In sand, fast growing microbes (for example, Achromobacter, Arthrobacter and Massilia) that utilise plantderived compounds had an advantage over slower growing microbes (Figure 3a). By the third generation, model plants grown in sand had rhizosphere microbiota dominated by bacterial opportunists as indicated by the abundance of the same OTUs in unplanted soil (Figure 3a and Supplementary Figure S4b). There was also a relative increase in Proteobacteria during the second and third generations that may reflect the disturbance caused by the selection system in sand (Figure 1). Crucially, the increase in Proteobacteria was much greater in unplanted sand in the second and third generations compared with plant rhizospheres (Supplementary Figure S4a). Thus even though diversity reduced substantially in the rhizosphere microbiota in generations two and three, plants still exerted a significant selection relative to soil. The increase in abundance of Proteobacteria, which are often fast growing, may be a signature of disturbance. Proteobacteria also increase in abundance in the human gut microbiota in patients infected by Clostridium difficile, which often follows antibiotic treatment, an effect reversed by faecal transplant (Hamilton et al., 2013). We hypothesise that the loss of diversity and increased proteobacterial abundance in our study was greater in unplanted sand than in plant rhizospheres because of stronger disruption. This suggests the initial soil inoculum, from which the microbial community comes, is more adapted to the rhizosphere environment than to poor carbon conditions that occurred in the unplanted sand control.

With regard to our second question, it is apparent that each plant selected a largely different rhizosphere microbiota in sand compared to compost from the initial microbial inoculum (Bawburgh soil), (Supplementary Figure S5). Thus plant type interacts with soil to affect selection of the microbiota. Furthermore, the collapse in diversity that occurred in the third generation of plants grown in sand did not occur in compost, as shown by the stable Shannon index of diversity (Supplementary Figures S4c and d). Exudates produced by roots are clearly important in plant selection of microbiota (Eilers et al., 2010), but a rich source of organic material in soil is critical for stability and maintenance of diversity.

Although compost helped maintain a stable community there was a remarkable invasion over plant generations of a fungal OTU that, based on ITS sequences, appears to be the pathogen $O$. brassicae or a close relative (Hartwright et al., 2010). This only 
occurred on its compatible host B. rapa (Figure 4). Intriguingly, there was also an increase in abundance of Massilia OTUs. Massilia is able to degrade chitin (Faramarzi et al., 2009; Adrangi et al., 2010) found in the fungus cell wall (James et al., 2006). It may also explain the repeated observation that Massilia is abundant in the rhizosphere (Bodenhausen et al., 2013) and found to be associated with soils colonised by pathogenic fungi like Fusarium and Rhizoctonia solani (Klein et al., 2013; Yin et al., 2013). Moreover, it forms at least commensal interactions with Pythium aphanidermatum, a phytopathogenic oomycete infecting cucumber seeds (Ofek et al., 2012). However, our data do not allow us to determine whether the occurrence of Olpidium and Massilia in Brassica rhizospheres at much higher abundance than any other microorganism reflects a trophic relationship between them or simply a common preference for Brassica roots.

The Arabidopsis microbiota in sand collapsed in diversity in the third generation and although it remained highly diverse in compost, it divided into three divergent groups with significantly different microbiota, suggesting stochastic variation (Supplementary Figure S2j) and possibly the occurrence of multiple stable states in the rhizosphere community (Sutherland, 1974). Swenson et al., 2000, conducted repeated replanting of Arabidopsis thaliana Landsberg erecta in compost for 16 generations, which led to significant divergence of plant growth particularly after 10 generations. In this case, plants were weighed at each generation and soil from the top $10 \%$ and bottom $10 \%$ of weights was used as a microbiota inoculum for each successive generation. Compost derived from the heaviest plants lead to enhanced growth in successive generations relative to compost from the lightest plants. In our study it was impractical to continue a detailed metagenomic analysis for 10-16 generations, but it suggests that repeated sub-culturing with plant selection may lead to very different microbiota becoming fixed with significant consequences for plant growth.

We believe that this is the first report using a multi-replicate system to study the influence of plants on the rhizosphere microbiota and its stability over plant generations. Using high-throughput sequencing methods it should now be possible to focus on the importance of rhizoplane and endosphere microbial communities using a similar experimental design. Future research should take into consideration that only by using a high number of biological replicates it is possible to use statistics to determine significance in studies of microbial ecology. Here we also show the power of selection experiment in rhizosphere enrichment with the isolation of plant growth promoting rhizobacteria species. We not only demonstrate their growth promoting potential but also show that these strains are very competitive in the rhizosphere and their influence on the plant is species specific. In order to successfully use microbial inocula in agricultural systems it is essential to identify microorganisms that promote the growth of plants of interest and be competitive against other soil microorganisms (Lugtenberg and Kamilova, 2009).

Overall, the experimental selection system we used has proved particularly powerful at demonstrating that plant type and soil composition interact to control the rhizosphere microbiota, and allowing us to separate the influence of soil type from that of initial inoculum. Plants select distinct microbiota but their composition and stability also depend on soil.

\section{Conflict of Interest}

The authors declare no conflict of interest.

\section{Acknowledgements}

The research was funded by Zuckerman Endowment Fund and UK Biotechnology and Biological Sciences Research Council (BBSRC) grant BB/K001868/1. We thank Darren Heavens (The Genome Analysis Centre) and John Walshaw (John Innes Centre) for sequencing and initial data analysis, Rob Green (John Innes Centre) for help with figures, Alison East (John Innes Centre) for help with the manuscript and Peter Sterk (EMBL-EBI) for data submission. Metagenomic sequence data have been submitted to the EMBL databases under accession PRJEB7318.

\section{References}

Adrangi S, Faramarzi MA, Shahverdi AR, Sepehrizadeh Z. (2010). Purification and characterization of two extracellular endochitinases from Massilia timonae. Carbohydr Res 345: 402-407.

Bent SJ, Forney LJ. (2008). The tragedy of the uncommon: understanding limitations in the analysis of microbial diversity. ISME J 2: 689-695.

Berg G, Smalla K. (2009). Plant species and soil type cooperatively shape the structure and function of microbial communities in the rhizosphere. FEMS Microbiol Ecol 68: 1-13.

Bertani G. (1951). Studies on lysogenesis. I. The mode of phage liberation by lysogenic Escherichia coli. J Bacteriol 62: 293-300.

Bodenhausen N, Horton MW, Bergelson J. (2013). Bacterial communities associated with the leaves and the roots of Arabidopsis thaliana. PLoS One 8: e56329.

Bressan M, Roncato MA, Bellvert F, Comte G, Haichar FZ, Achouak W et al. (2009). Exogenous glucosinolate produced by Arabidopsis thaliana has an impact on microbes in the rhizosphere and plant roots. ISME J 3: 1243-1257.

Buee M, Reich M, Murat C, Morin E, Nilsson RH, Uroz S et al. (2009). 454 Pyrosequencing analyses of forest soils reveal an unexpectedly high fungal diversity. New Phytol 184: 449-456.

Bulgarelli D, Rott M, Schlaeppi K, Ver Loren van Themaat E, Ahmadinejad N, Assenza F et al. (2012). Revealing structure and assembly cues for Arabidopsis rootinhabiting bacterial microbiota. Nature 488: 91-95.

Bulgarelli D, Schlaeppi K, Spaepen S, van Themaat EVL, Schulze-Lefert P. (2013). Structure and functions of the 
bacterial microbiota of plants. Annu Rev Plant Biol 64: 807-838.

Cardinale M, Brusetti L, Quatrini P, Borin S, Puglia AM, Rizzi A et al. (2004). Comparison of different primer sets for use in automated ribosomal intergenic spacer analysis of complex bacterial communities. Appl Environ Microbiol 70: 6147-6156.

Demoling F, Figueroa D, Bååth E. (2007). Comparison of factors limiting bacterial growth in different soils. Soil Biol and Biochem 39: 2485-2495.

Dennis PG, Miller AJ, Hirsch PR. (2010). Are root exudates more important than other sources of rhizodeposits in structuring rhizosphere bacterial communities? FEMS Microbiol Ecol 72: 313-327.

Edgar RC. (2010). Search and clustering orders of magnitude faster than BLAST. Bioinformatics 26: 2460-2461.

Eilers KG, Lauber CL, Knight R, Fierer N. (2010). Shifts in bacterial community structure associated with inputs of low molecular weight carbon compounds to soil. Soil Biol Biochem 42: 896-903.

Faramarzi M.A. FM, Tabatabaei Yazdi M, Adrangi S, Jamialahmadi K, Tasharrofi N, Azizmohseni F. (2009). Optimization of cultural condtions for production of chitinase by a soil isolate of Massilia timonae. Biotechnology 8: 99-93.

Fisher MM, Triplett EW. (1999). Automated approach for ribosomal intergenic spacer analysis of microbial diversity and its application to freshwater bacterial communities. Appl Environ Microbiol 65: 4630-4636.

Griffiths RI, Whiteley AS, O'Donnell AG, Bailey MJ. (2000). Rapid method for coextraction of DNA and RNA from natural environments for analysis of ribosomal DNAand rRNA-based microbial community composition. Appl Environ Microbiol 66: 5488-5491.

Hamady M, Knight R. (2009). Microbial community profiling for human microbiome projects: tools, techniques, and challenges. Genome Res 19: 1141-1152.

Hamilton MJ, Weingarden AR, Unno T, Khoruts A, Sadowsky MJ. (2013). High-throughput DNA sequence analysis reveals stable engraftment of gut microbiota following transplantation of previously frozen fecal bacteria. Gut Microbes 4: 125-135.

Hartwright LM, Hunter PJ, Walsh JA. (2010). A comparison of Olpidium isolates from a range of host plants using internal transcribed spacer sequence analysis and host range studies. Fungal Biol 114: 26-33.

Hawes MC, Gunawardena U, Miyasaka S, Zhao X. (2000). The role of root border cells in plant defense. Trends Plant Sci 5: 128-133.

Hilton S, Bennett AJ, Keane G, Bending GD, Chandler D, Stobart R et al. (2013). Impact of shortened crop rotation of oilseed rape on soil and rhizosphere microbial diversity in relation to yield decline. PLoS One 8: e59859.

Huson DH, Richter DC, Mitra S, Auch AF, Schuster SC. (2009). Methods for comparative metagenomics. BMC Bioinform 10: S12.

James TY, Letcher PM, Longcore JE, Mozley-Standridge SE, Porter D, Powell MJ et al. (2006). A molecular phylogeny of the flagellated fungi (Chytridiomycota) and description of a new phylum (Blastocladiomycota). Mycologia 98: 860-871.

Jones DL, Nguyen C, Finlay RD. (2009). Carbon flow in the rhizosphere: carbon trading at the soil-root interface. Plant Soil 321: 5-33.
Kinkel LL, Bakker MG, Schlatter DC. (2011). A coevolutionary framework for managing diseasesuppressive soils. Annu Rev Phytopathol 49: 47-67.

Klein E, Ofek M, Katan J, Minz D, Gamliel A. (2013). Soil suppressiveness to fusarium disease: shifts in root microbiome associated with reduction of pathogen root colonization. Phytopathology 103: 23-33.

Knight R, Jansson J, Field D, Fierer N, Desai N, Fuhrman JA et al. (2012). Unlocking the potential of metagenomics through replicated experimental design. Nat Biotechnol 30: 513-520.

Lambert B, Meire P, Joos H, Lens P, Swings J. (1990). Fastgrowing, aerobic, heterotrophic bacteria from the rhizosphere of young sugar beet plants. Appl Environ Microbiol 56: 3375-3381.

Lanfranco L, Young JP. (2012). Genetic and genomic glimpses of the elusive arbuscular mycorrhizal fungi. Curr Opin Plant Biol 15: 454-461.

Lauber CL, Hamady M, Knight R, Fierer N. (2009). Pyrosequencing-based assessment of soil $\mathrm{pH}$ as a predictor of soil bacterial community structure at the continental scale. Appl Environ Microbiol 75: 5111-5120.

Lebreton L, Gosme M, Lucas P, Guillerm-Erckelboudt A-Y, Sarniguet A. (2007). Linera relationship bewteen Gaeumannomyces graminis var. tritici (Ggt) gentypic frequencies and diverse severity on wheat roots in the field. Environ Microbiol 9: 492-499.

Lugtenberg B, Kamilova F. (2009). Plant-growth-promoting rhizobacteria. Annu Rev Microbiol 63: 541-556.

Lundberg DS, Lebeis SL, Paredes SH, Yourstone S, Gehring J, Malfatti S et al. (2012). Defining the core Arabidopsis thaliana root microbiome. Nature 488: 86-90.

Manter DK, Delgado JA, Holm DG, Stong RA. (2010). Pyrosequencing reveals a highly diverse and cultivar-specific bacterial endophyte community in potato roots. Microb Ecol 60: 157-166.

Mendes R, Kruijt M, de Bruijn I, Dekkers E, van der Voort M, Schneider JH et al. (2011). Deciphering the rhizosphere microbiome for disease-suppressive bacteria. Science 332: 1097-1100.

Murashige T, Skoog F. (1962). A revised medium for rapid growth and bioassays with tobaco tissue cultures. Physiol Plant 15: 473-497.

Ngugi DK, Stingl U. (2012). Combined analyses of the ITS loci and the corresponding $16 \mathrm{~S}$ rRNA genes reveal high micro- and macrodiversity of SAR11 populations in the Red Sea. PLoS One 7: e50274.

Nuccio EE, Hodge A, Pett-Ridge J, Herman DJ, Weber PK, Firestone MK. (2013). An arbuscular mycorrhizal fungus significantly modifies the soil bacterial community and nitrogen cycling during litter decomposition. Environ Microbiol 15: 1870-1881.

O'Reilly T, Niven DF. (1986). Tryptone-yeast extract broth as a culture medium for Haemophilus pleuropneumoniae and Haemophilus parasuis to be used as challenge inocula. Can J Vet Res 50: 441-443.

Ofek M, Hadar Y, Minz D. (2012). Ecology of root colonizing Massilia (Oxalobacteraceae). PLoS One 7: e40117.

Osborne CA, Zwart AB, Broadhurst LM, Young AG, Richardson AE. (2011). The influence of sampling strategies and spatial variation on the detected soil bacterial communities under three different landuse types. FEMS Microbiol Ecol 78: 70-79.

Poole PS, Blyth A, Reid CJ, Walters K. (1994a). myoInositol catabolism and catabolite regulation in 
Rhizobium leguminosarum bv. viciae. Microbiology 140: $2787-2795$.

Poole PS, Schofield NA, Reid CJ, Drew EM, Walshaw DL. (1994b). Identification of chromosomal genes located downstream of $d c t \mathrm{D}$ that affect the requirement for calcium and the lipopolysaccharide layer of Rhizobium leguminosarum. Microbiology 140: 2797-2809.

$\mathrm{R}$ Development Core Team (2008). R: A Language and Environment for Statistical Computing. R Foundation for Statistical Computing: Vienna, Austria. http:// www.R-project.org.

Sharma S, Aneja MK, Mayer J, Munch JC, Schloter M. (2005). Characterization of bacterial community structure in rhizosphere soil of grain legumes. Microb Ecol 49: $407-415$.

Smith CJ, Danilowicz BS, Clear AK, Costello FJ, Wilson B, Meijer WG. (2005). T-Align, a web-based tool for comparison of multiple terminal restriction fragment length polymorphism profiles. FEMS Microbiol Ecol 54: $375-380$.

Sonderby IE, Hansen BG, Bjarnholt N, Ticconi C, Halkier BA, Kliebenstein DJ. (2007). A systems biology approach identifies a R2R3 MYB gene subfamily with distinct and overlapping functions in regulation of aliphatic glucosinolates. PLoS One 2: e1322.

Sutherland JP. (1974). Multiple stable states in natural communities. Am Nat 108: 859-873.

Swenson W, Wilson DS, Elias R. (2000). Artificial ecosystem selection. Proc Natl Acad Sci USA 97: 9110-9114.

Taghavi S, Garafola C, Monchy S, Newman L, Hoffman A, Weyens $N$ et al. (2009). Genome survey and characterization of endophytic bacteria exhibiting a beneficial effect on growth and development of poplar trees. Appl Environ Microbiol 75: 748-757.

Terpolilli JJ, Hood GA, Poole PS. (2012). What determines the efficiency of N(2)-fixing Rhizobium-legume symbioses? Adv Microb Physiol 60: 325-389.

Turner TR, James EK, Poole PS. (2013a). The plant microbiome. Genome Biol 14: 209.

Turner TR, Ramakrishnan K, Walshaw J, Heavens D, Alston M, Swarbreck D et al. (2013b). Comparative metatranscriptomics reveals kingdom level changes in the rhizosphere microbiome of plants. ISME $J$ 7: 2248-2258.

Udvardi M, Poole PS. (2013). Transport and metabolism in legume-rhizobia symbioses. Annu Rev Plant Biol 64: 781-805.

Van der Ent S, Van Wees SC, Pieterse CM. (2009). Jasmonate signaling in plant interactions with resistance-inducing beneficial microbes. Phytochemistry 70: 1581-1588.

Wooley JC, Godzik A, Friedberg I. (2010). A primer on metagenomics. PLoS Comput Biol 6: e1000667.

Wright ES, Yilmaz LS, Noguera DR. (2012). DECIPHER, a search-based approach to chimera identification for 16S rRNA sequences. Appl Environ Microbiol 78: 717-725.

Yin C, Hulbert SH, Schroeder KL, Mavrodi O, Mavrodi D, Dhingra A et al. (2013). Role of bacterial communities in the natural suppression of Rhizoctonia solani bare patch disease of wheat (Triticum aestivum L.). Appl Environ Microbiol 79: 7428-7438.

Yuan H, Ge T, Chen C, O'Donnell AG, Wu J. (2012). Significant role for microbial autotrophy in the sequestration of soil carbon. Appl Environ Microbiol 78: $2328-2336$.

Supplementary Information accompanies this paper on The ISME Journal website (http://www.nature.com/ismej) 